\title{
Hydrophilic Multitemplate Molecularly Imprinted Biopolymers Based on a Green Synthesis Strategy for Determination of B-Family Vitamins
}

\author{
Abbas Ostovan, ${ }^{\dagger}$ Mehrorang Ghaedi, ${ }^{*}{ }^{\ddagger}$ Maryam Arabi, ${ }^{\ddagger}{ }^{\dagger}$ Qian Yang, ${ }^{\S}$ Jinhua Li, ${ }^{\S}$ and Lingxin Chen ${ }^{*}, \S(0)$ \\ ${ }^{\dagger}$ Department of Chemistry, Kerman Branch, Islamic Azad University, Kerman, Iran \\ ${ }^{\ddagger}$ Chemistry Department, Yasouj University, Yasouj 75918-74831, Iran \\ ${ }^{\S}$ Key Laboratory of Coastal Environmental Processes and Ecological Remediation, Yantai Institute of Coastal Zone Research, Chinese \\ Academy of Sciences, Yantai 264003, China
}

Supporting Information

\begin{abstract}
A novel green synthesis strategy was proposed for preparation of multitemplate molecularly imprinted biopolymers (mt-MIBP) in aqueous media with less consumption of organic solvents, which were subsequently used as sorbents of ultrasound-assisted dispersive solid-phase extraction (d-SPE) for simultaneous recognition and efficient separation of B-family vitamins in juice samples, followed by high performance liquid chromatography (HPLC) determination. The obtained mt-MIBP was fully characterized by SEM, FT-IR, TEM, and BET. It offered high binding capacity, good selectivity, and fast dynamics toward all the templates. Involved parameters in the d-SPE efficiency such as mt-MIBP mass, sonication time, and eluting/ washing solvents' types and volumes were concurrently investigated by central composite design with rapidity and reliability. Under the optimum conditions, the developed mt-MIBP-d-SPE-HPLC method exhibited wide linear range, low limits of detection and quantification (LOQs) within $1.2-5.5 \mu \mathrm{g} \mathrm{L}^{-1}$ and $4.0-18.4 \mu \mathrm{g} \mathrm{L}^{-1}$, respectively, and appropriate repeatability (relative standard deviation values below $4.2 \%, n=4$ ). The high selectivity of this method makes it suitable for successful monitoring of vitamins in juice samples with satisfactory recoveries of $75.8-92.7 \%, 81.1-92.5 \%$, and $84.7-93.8 \%$ for vitamins riboflavin $\left(B_{2}\right)$, nicotinamide $\left(B_{3}\right)$, and pyridoxine $\left(B_{6}\right)$, respectively. The present study implied highly promising perspectives of water-compatible eco-friendly mt-MIBP for highly effective multiresidue analysis in complicated matrixes.
\end{abstract}

KEYWORDS: molecularly imprinted polymers, multitemplate imprinting, green synthesis, dispersive solid-phase extraction, experimental design, vitamins

\section{INTRODUCTION}

The dramatic increase in the variety of analytical instrumentation and emerging hyphenated chromatographic techniques such as gas chromatography-mass spectrometry (GC-MS), high performance liquid chromatography-fluorescence spectrometry (HPLC-FL), and so on allows determination of most compounds in various matrixes including environmental, food, and biological samples. Nevertheless, even using highly sensitive and selective detection techniques like MS, direct injection of crude extracts is usually not recommended because of the complexity of most sample matrixes, which can inhibit or enhance the analyte's ionization or seriously pollute the separator/detector. ${ }^{1}$ Accordingly, effective sample preparation steps before instrumental analysis are critical to identify and ultimately provide accurate quantification of analytes. ${ }^{2,3}$ The significant aims of sample preparation are elimination of matrix interfering agents and/or analyte preconcentration, and ultimately conversion of the target compounds into a more appropriate form for detection or preliminary separation (if needed). For this regard, solid-phase extraction (SPE), solid-

Received: November 16, 2017

Accepted: January 4, 2018

Published: January 4, 2018 
phase microextraction (SPME), liquid-liquid microextraction (LLME), matrix solid-phase dispersion (MSPD), and so on have been applied for analytes preconcentration/separation and matrix elimination. Among available procedures, a considerable interest has been devoted to dispersive SPE (d-SPE), which is based on SPE and the sorbents are directly added into the sample solution without conditioning. Compared with general SPE, the d-SPE not only can avoid the tedious treatment of the conventional adsorbents and the easy blockage of the adsorption column but also has higher repeatability and less solvent consumption. ${ }^{4}$ However, d-SPE also suffers from lack of selectivity, easily leading to undesirable consequences and lower detection sensitivity.

Molecularly imprinted polymers (MIPs), as synthetic receptors with artificially generated recognition sites for target compounds based on memory of the shape, size, and functional groups of the template, have received extensive attention as selective sorbents for effective separation, purification, and enrichment of trace targets in complicated matrixes. ${ }^{5-7}$ Meanwhile, surface imprinting based MIPs have been increasingly prepared, showing complete template removal, good site accessibility, favorable mass-transfer, and binding capacity. ${ }^{6}$ For the imprinting strategy, various supports (core) especially with large surface area and high porosity are available, such as carbon nanotubes, graphene oxide, silica nanoparticles, quantum dots, and carbon spheres (CSs). ${ }^{6,8}$ Due to superior properties including cost-effective natural precursor, environmentally friendly synthesis process, functionally sustainable micro/nanostructure, excellent biocompatibility and chemical inertness, nonimmunogenic, and so on, ${ }^{8,9}$ CS has attracted wide concerns as a versatile core support for surface imprinting. ${ }^{10-12}$ Accordingly, we have been encouraged to use glucose as biobased precursor for CS preparation by a simple and facile hydrothermal route. However, to the best of our knowledge, in all CS based MIPs reported, organic solvents and reagents have been utilized within the surface imprinting process. For instance, dibenzothiophene imprinted CS@MIPs often suffer from consumption of organic solvents and reagents and matrix interferences such as proteins and lipids which are easily adsorbed on the surface of acrylic based MIPs and lead to critical restriction of mass transfer of small template and serious decrease of extraction efficiency in biological samples. ${ }^{10-12}$ To overcome the obstacles in the line of green chemistry, researchers are encouraged to use natural polymers which are known as a beneficial alternative due to impressive advantages such as eco-friendliness, less hazardous synthesis, and waste prevention. Natural polymers and their derivatives possess mechanically stable three-dimensional structures, in which the template molecule is entrapped at specific molecular recognition sites based on adoptability of shape, size, and functional groups which subsequently are imprinted into polymeric structures. $^{13}$

On the other hand, it has been largely demonstrated that small organic analytes mostly fail to show selective specific binding in aqueous media compared to organic solvents used for the MIPs preparation. It is probably related to the least shrinking and/or swelling of the MIP structure exposed to the porogen used. ${ }^{14}$ In addition, hydrophobicity of the generic MIPs and hydrophobic interferences are greatly increased in biological samples in comparison to in organic solvents and lead to the appearance of significant nonspecific hydrophobic interactions between MIPs and templates. ${ }^{15}$ So far, a variety of strategies have been developed to address the problems, such as using water-methanol or pure aqueous buffer during MIP synthesis, ${ }^{16,17}$ using special polymerizable monomers, ${ }^{18,19}$ and grafting hydrophilic polymer layers onto MIP surfaces ${ }^{20,21}$ or hydrophilic functional groups onto the MIP surfaces by chemical modification. ${ }^{22}$ However, toxic substances and/or organic solvents are still needed. To reduce usage of chemical reagents and waste prevention, we purposed to employ chitosan as a versatile biosubstance to form an imprinting network on the surface of CSs at aqueous media, because of its unique properties including low cost, nontoxicity, biodegradability and biocompatibility, and high ability to polymerize in mild condition.

Another important issue involved in analytical methods is reasonable separation and detection of a series of similar analytes present in different complicated samples. On the other hand, the single template-imprinted cavity produced in common MIPs can cause considerable constraint for concurrent extraction of several analytes. Alternatively, multitemplate-recognition networks using more than one compound as templates in a single MIP format have been reported. ${ }^{23-25}$ The attainment to short extraction time as well as cost-effective analyses for multiple analytes in complex matrixes possessing much interferent is a worthwhile task. So, we expected to synthesize multitemplate MIPs that can simultaneously recognize and bind a group of biologically important compounds, such as vitamins. Vitamins are considered as vital nutrients for controlling the human normal health and growth in limited amounts. ${ }^{26}$ So that, the dosage level in food may be as low as a few micrograms per $100 \mathrm{~g}$. Also, accompaniment by some potentially adverse compounds with relatively similar chemical properties can remarkably affect a vitamin's availability. ${ }^{27}$ Unfortunately, influential preconcentration of water-soluble vitamins via nonpolar columns of SPE is not feasible and results in no quantitative retention and consequently considerable reduction in extraction efficiency. ${ }^{26-28}$ So, simultaneous, quantitative analysis of vitamins especially the B-family in foodstuff is of important significance.

Therefore, in this work, through a simple, time-saving, and facile green synthesis strategy with low consumption of organic solvent, we prepared novel hydrophilic multitemplate molecularly imprinted biopolymers (mt-MIBP) by using the biosubstance of chitosan to form an imprinting network on the CS surface in aqueous media and at ambient temperature, for simultaneous selective recognition and extraction of Bfamily vitamins. Three typical vitamins including riboflavin (vitamin $\mathrm{B}_{2}$ ), nicotinamide (vitamin $\mathrm{B}_{3}$ ), and pyridoxine (vitamin $\mathrm{B}_{6}$ ) were chosen as template molecules, and the well characterized mt-MIBP was employed as d-SPE sorbents, followed by HPLC-UV determination. Central composite design (CCD) and response surface were utilized to quickly reliably assist finding optimum extraction conditions. The mtMIBP based d-SPE coupled with HPLC with the aid of experimental design was developed, validated, and successfully applied for simultaneous separation and determination of Bfamily vitamins in orange juice samples.

\section{EXPERIMENTAL SECTION}

2.1. Materials and Instruments. Glucose, 1 -hexanesulfonic acid sodium salt, riboflavin (vitamin $B_{2}$ ), nicotinamide (vitamin $B_{3}$ ), pyridoxine (vitamin $\mathrm{B}_{6}$ ), ascorbic acid (vitamin $\mathrm{C}$ ), thiamine (vitamin $\left.\mathrm{B}_{1}\right)$, acetic acid, and ammonium hydroxide $\left(\mathrm{NH}_{3} \cdot \mathrm{H}_{2} \mathrm{O}\right)$ were purchased from Merck (Darmstadt, Germany). Methanol, hexane, ethanol, acetone, and acetonitrile (liquid chromatography grade) were 


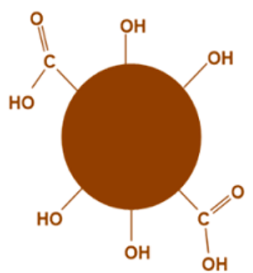<smiles>OC[C@H]1O[C@H](O)[C@@H](O)[C@H](O)[C@H]1O</smiles>

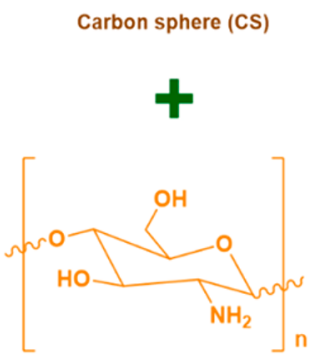

Chitosan

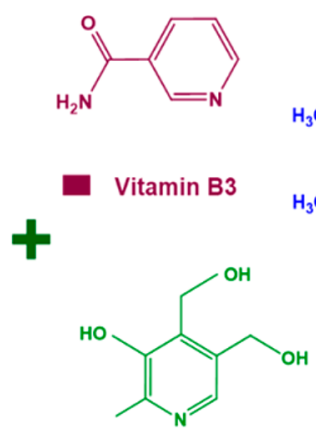

$\triangle$ Vitamin B6<smiles>Cc1cc2c(cc1C)N(C[C@H](O)[C@H](O)[C@H](O)CO)C1NC(=O)NC(=O)C1=N2</smiles>

Vitamin B2
Deionized water

Acetic Acid

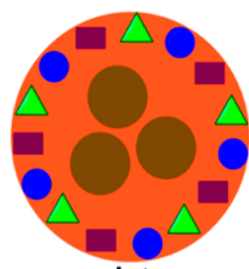

Template removal $\quad$ Rebinding

mt-MIBP added, allowing forming precomplexes between chitosan and templates at room temperature for $2 \mathrm{~h}$. At the next step, $0.25 \mathrm{~g}$ of CS was transferred to the above solution, followed by stirring vigorously for $24 \mathrm{~h}$ at ambient temperature. Afterward, $50 \mathrm{~mL}$ of $1 \mathrm{~mol}$ $\mathrm{L}^{-1} \mathrm{NaOH}$ solution was added rapidly into the resultant solution and kept stirring for $3 \mathrm{~h}$. Afterward, the prepared product was collected by centrifugation, followed by several rinsing with deionized water until reaching the neutral $\mathrm{pH}$ of 7.0 and was redispersed in $0.5 \mathrm{~mol} \mathrm{~L}^{-1}$ $\mathrm{H}_{2} \mathrm{SO}_{4}$ for 5 min under ultrasonic irradiation. Finally, mt-MIBP was attained after eluting vitamins repeatedly with methanol-deionized water $(50: 50, \mathrm{v} / \mathrm{v})$ until template molecules were removed completely. The basic preparation procedure is schematically shown in Figure 1. The nonimprinted polymers (NIPs) were synthesized with the same procedure except in the absence of templates.

2.3. Adsorption Test. Study of adsorption was carried out by suspending $40 \mathrm{mg}$ of mt-MIBP or NIBP into $10 \mathrm{~mL}$ of a solution of three vitamins at varied initial concentration $\left(50.0-600.0 \mathrm{mg} \mathrm{L}^{-1}\right)$. After magnetic stirring at $25{ }^{\circ} \mathrm{C}$ for $20 \mathrm{~min}$, the sorbents were collected by centrifugation, and residual concentrations of vitamins were determined by HPLC. Also, the binding kinetics of mt-MIBP and NIBP were analyzed at specific time intervals from 1 to $40 \mathrm{~min}$ following addition of $40 \mathrm{mg}$ of $\mathrm{mt}-\mathrm{MIBP} / \mathrm{NIBP}$ to the water solution of each vitamin $\left(5.0 \mathrm{mg} \mathrm{L}^{-1}\right)$. Then, the solution was filtered and their residual concentrations in supernatant solution were determined by HPLC.

A $40 \mathrm{mg}$ portion of mt-MIBP was suspended in $10.0 \mathrm{~mL}$ of aqueous solution of interfering reagent at a concentration of $250.0 \mathrm{mg} \mathrm{L}^{-1}$. In next stage, these solutions were sonicated for $20 \mathrm{~min}$ load analytes by solid phase, and subsequently, mt-MIBP was isolated by centrifugation, followed by individual monitoring of the nonaccumulated contents of components in supernatant solutions.

2.4. Preparation of Calibration Standards and d-SPE Procedure of Vitamins from Orange Juice Samples. Standard stock solutions of B-family vitamins were prepared in deionized water at $1.0 \mathrm{mg} \mathrm{mL} \mathrm{m}^{-1}$ and were stored in a refrigerator at $4{ }^{\circ} \mathrm{C}$. Subsequently, the working solutions were obtained by the appropriate dilution with deionized water. Orange juice samples were purchased from a local supermarket in Shiraz and stored at $4{ }^{\circ} \mathrm{C}$ before use, and all samples were simply filtered through Whatman paper No. 42 before treatment. 


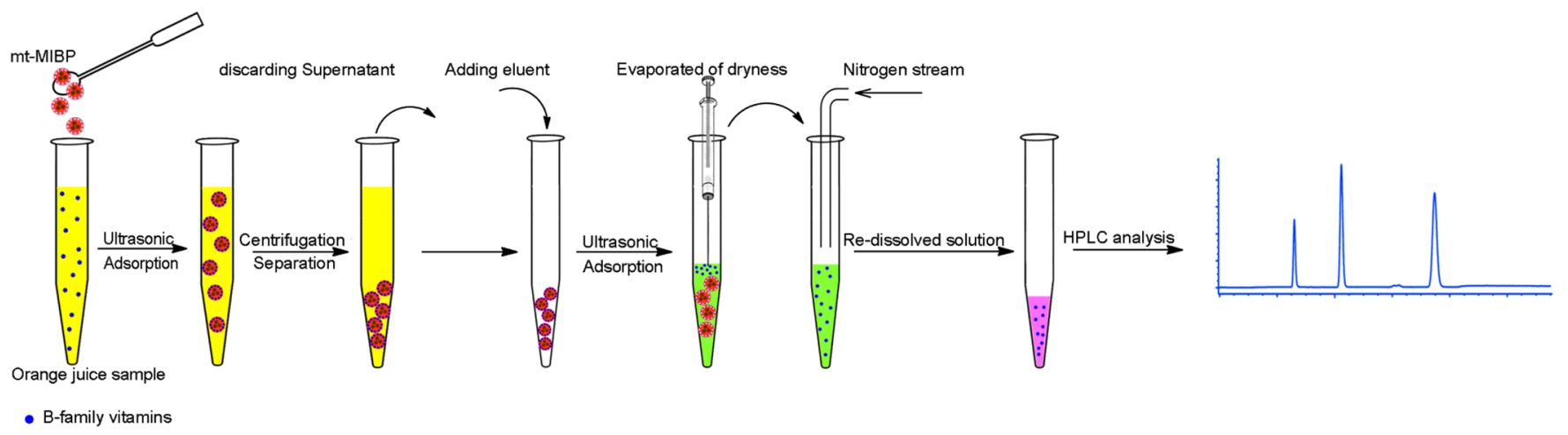

Figure 2. Schematic illustration of mt-MIBP-d-SPE-HPLC-UV procedure.

Figure 2 illustrates the mt-MIBP based d-SPE process. Under optimized conditions, $40 \mathrm{mg}$ of mt-MIBP was dispersed in $10 \mathrm{~mL}$ of orange juice with $\mathrm{pH}$ adjusted at 11.0. After ultrasonication for 20 $\mathrm{min}$, the mt-MIBP was separated with a centrifuge $(6000 \mathrm{rpm}, 10 \mathrm{~min})$ and subsequently washed with $2 \mathrm{~mL}$ of hexane for elimination of potential interfering agents. Subsequently, analytes of interest were eluted by redispersion of mt-MIBP in $1 \mathrm{~mL}$ of eluting solvent consisting of methanol-acetonitrile-deionized water-ammonium hydroxide (30:30:30:10, v/v) for three times. All eluents were merged together, followed by evaporation to dryness under nitrogen, and the residue was subsequently dissolved in $50 \mu \mathrm{L}$ of mobile phase and ready for HPLC analysis.

\section{RESULTS AND DISCUSSION}

3.1. Preparation and Characterization of mt-MIBP. The schematic preparation procedure of core-shell structured mt-MIBP is represented in Figure 1. As seen, glucose was employed as carbon natural resource to prepare CS by a hydrothermal route. The criterion for selection of glucose is according to green and sustainable chemistry, to avoid or use less dangerous chemicals and to intensify waste prevention. Glucose is versatile in terms of nontoxicity, environmental friendliness, good water-solubility and biocompatibility, easy availability, and wide practicality. Especially, glucose can be readily converted into an interesting CS structure. Moreover, the obtained CS is decorated with abundant surface polar oxygenated functionalities still remaining from the original glucose, which can offer efficient sites for further functionalization of chitosan. ${ }^{29}$ In the next stage, a layer of chitosan was deposited onto CS through a facile, one-step, and green process at room temperature in the vicinity of B-family vitamins as templates. Chitosan was dissolved and protonated significantly in acidic media, whereas CS induced negative charge and ultimately resulted in strong electrostatic interactions between $\mathrm{CS}$ and chitosan. ${ }^{30}$ Then, additional $\mathrm{NaOH}$ solution (i.e., basic condition) made chitosan precipitate onto the surface of CS to form mt-MIBP. ${ }^{31}$ Chitosan was chosen as the natural functional monomer, based on its three unique merits: (1) It has multifunctional structures $\left(-\mathrm{OH},-\mathrm{NH}_{2},-\mathrm{O}-\right.$ groups $)$ which could considerably interact with templates through hydrogen bonds in aqueous solution and could ultimately eliminate the by-effects of water as well as sustain the formation of a monomer-template complex. (2) Chitosan can easily crosslink with ionic interactions by a cheap inorganic agent (sulfuric acid) in mild conditions over short time. ${ }^{32,33}$ (3) Chitosan is hydrophilic in nature and is qualified as desirable material for mass transfer of water-soluble analytes from sample solutions to mt-MIBP. Meanwhile, B-family vitamins could be chosen as proper template analytes in terms of their analysis necessity due to proceeding the crucial metabolic reactions in the human body, their multifunctional structures leading to versatile and influential interactions with chitosan, and their large water miscibility ensuring these analytes to become ideal candidates for the proposed synthesis route. After templates removal by rinsing with methanol-deionized water, the selective cavities based on size, shape, and functional groups of templates were produced in the mt-MIBP structure.

The factors possibly influencing the preparation of mt-MIBP generally include types, amounts and ratios of template molecules, functional monomers, porogens, and cross-linkers, and preparation methods/strategies. ${ }^{6}$ A related optimization process is described in the Supporting Information, and the optimal preparation conditions are given in section 2.2. Although optimizing yields in chemical synthesis is important, other issues also need to be addressed, e.g., reducing the use of organic solvents, thereby decreasing pollution, which is one important goal of sustainable chemistry. There are several procedures for synthesis of MIPs, but most of them are not based on the green chemistry, so it is urgently demanded to develop eco-friendly, efficient, versatile, clean synthesis and less polluting methods. The selection of biobased substance at the service of imprinting technology is a suitable choice in agreement with green chemistry. Therefore, herein, a novel hydrophilic mt-MIBP with multiple recognition sites was obtained by using the biosubstance of chitosan to form an imprinting network on the CS surface in aqueous media without organic solvent consumption. Then, the mt-MIBP was well characterized as below.

Figure 3 shows FT-IR spectra of CS and mt-MIBP. A strong peak of hydroxyl at $3404 \mathrm{~cm}^{-1}$ was possibly related to the presence of $\mathrm{O}-\mathrm{H}$ groups on the CS surface (curve b). Notably, the characteristic bands at 1704 and $1619 \mathrm{~cm}^{-1}$ (together with the band at $1510 \mathrm{~cm}^{-1}$ ) could be assigned to the $\mathrm{C}=\mathrm{O}$ and $\mathrm{C}=\mathrm{C}$ vibrations, ${ }^{34}$ respectively. The bands around 1157 and $1398 \mathrm{~cm}^{-1}$ could be, respectively, attributed to the $\mathrm{C}-\mathrm{O}$ stretching and $\mathrm{O}-\mathrm{H}$ bending vibrations. The absorption peaks at 1630 and $1154 \mathrm{~cm}^{-1}$ in mt-MIBP were related to the bending vibration of $\mathrm{NH}_{2}$ and $\mathrm{C}-\mathrm{N}$ stretching vibration, 35 respectively, demonstrating that chitosan was deposited onto the CS.

The SEM/TEM images are considered as beneficial tools for identification of shape and surface morphology. As seen from Figures S1 and S2, the mt-MIBP had a relatively uniform structure with a regular spherical morphology with nanoscale diameter. And mt-MIBP possessed a low degree of agglomeration (Figure S1) which facilitated mass transfer and fast sorption kinetics. In addition, a velvety surface and low 


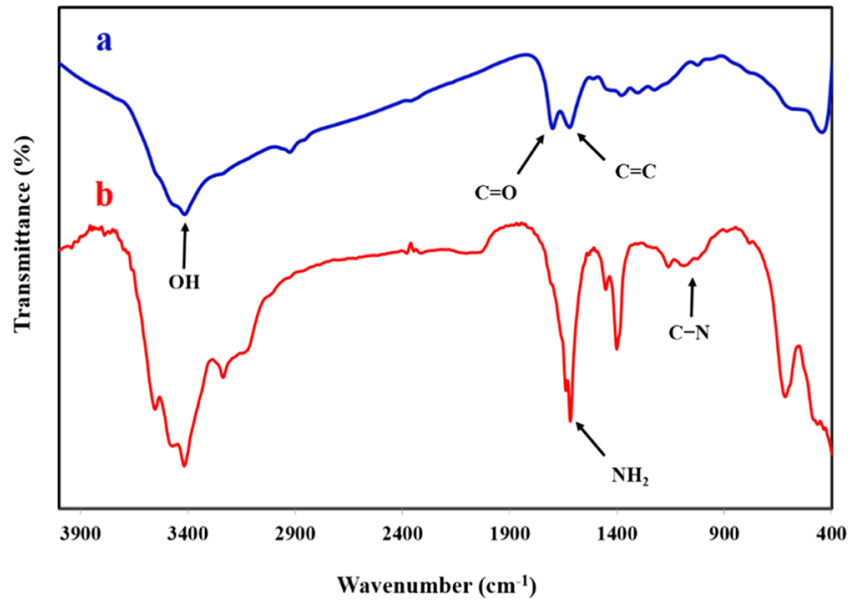

Figure 3. FT-IR spectra of (a) CS and (b) mt-MIBP.

dimension of mt-MIBP (Figure S2) easily led to complete template removal and subsequently large adsorption capacity. As seen, several black CSs existed in the internal surrounded by the chitosan layer, which revealed the CS particles were fully coated by the chitosan network. The distinct core-shell structure could account for rapid mass transfer and sorption/ desorption processes (Figure S2). Moreover, as shown in Figure S3, the statistic hydration particle size distribution further confirmed the mt-MIBP had homogeneous size distribution with a mean diameter of about $300 \mathrm{~nm}$. These observations provided powerful evidence of successful synthesis of mt-MIBP beads.

BET analysis provides precise specific surface area evaluation of prepared materials by nitrogen adsorption measured as a function of relative pressure at $-196{ }^{\circ} \mathrm{C}$. As seen in Figure S4, this kind of adsorption could be classified as a typical type IV isotherm which explained the formation of multilayer and also showed large deviation from the Langmuir model. The specific surface area of mt-MIBP was attained as $77.63 \mathrm{~m}^{2} \mathrm{~g}^{-1}$, which is much larger than that of NIP $\left(27.82 \mathrm{~m}^{2} \mathrm{~g}^{-1}\right)$ and is easily attributed to the template functioning, as well as, the average pore diameter of mt-MIBP was $6.46 \mathrm{~nm}$ higher than that of NIP $(2.76 \mathrm{~nm})$. Although the specific area of MIBP is lower than that reported for traditionally prepared multitemplate MIPs by Madikizela et al. ${ }^{36}$ and by Dai et al., ${ }^{37}$ our synthesis procedure is time-saving, simpler, more easily controllable, and more importantly, organic solvent is free, which is superior to the use of toluene. ${ }^{36,37}$

3.2. Adsorption Properties of the mt-MIBP for Vitamins. The adsorption kinetics and isotherms of $B_{2}, B_{3}$, and $\mathrm{B}_{6}$ onto the mt-MIBP and NIP were investigated. As presented in Figure $4 \mathrm{a}$, both imprinted and nonimprinted composites displayed a fast adsorption rate, while raising the extraction time had a positive trend with adsorption capacity and almost reached equilibrium after $20 \mathrm{~min}$ for all of the three different templates. It took 7.5 times shorter to reach adsorption saturation than that of acrylic-based surface imprinting MIPs, ${ }^{38}$ which could be attributed to the fact that the noticeable accessibility to the specific binding sites and subsequently quick diffusion of target analytes into them. In addition, adsorption equilibrium of analytes on NIP were faster than that on mt-MIBP, most likely due to the difference in their binding sites, while respective NIP only had one type of nonspecific binding sites which completely differed from mtMIBP. The thin and uniform imprinted chitosan shells of mtMIBP and NIP offered superior mass transfer, overcoming
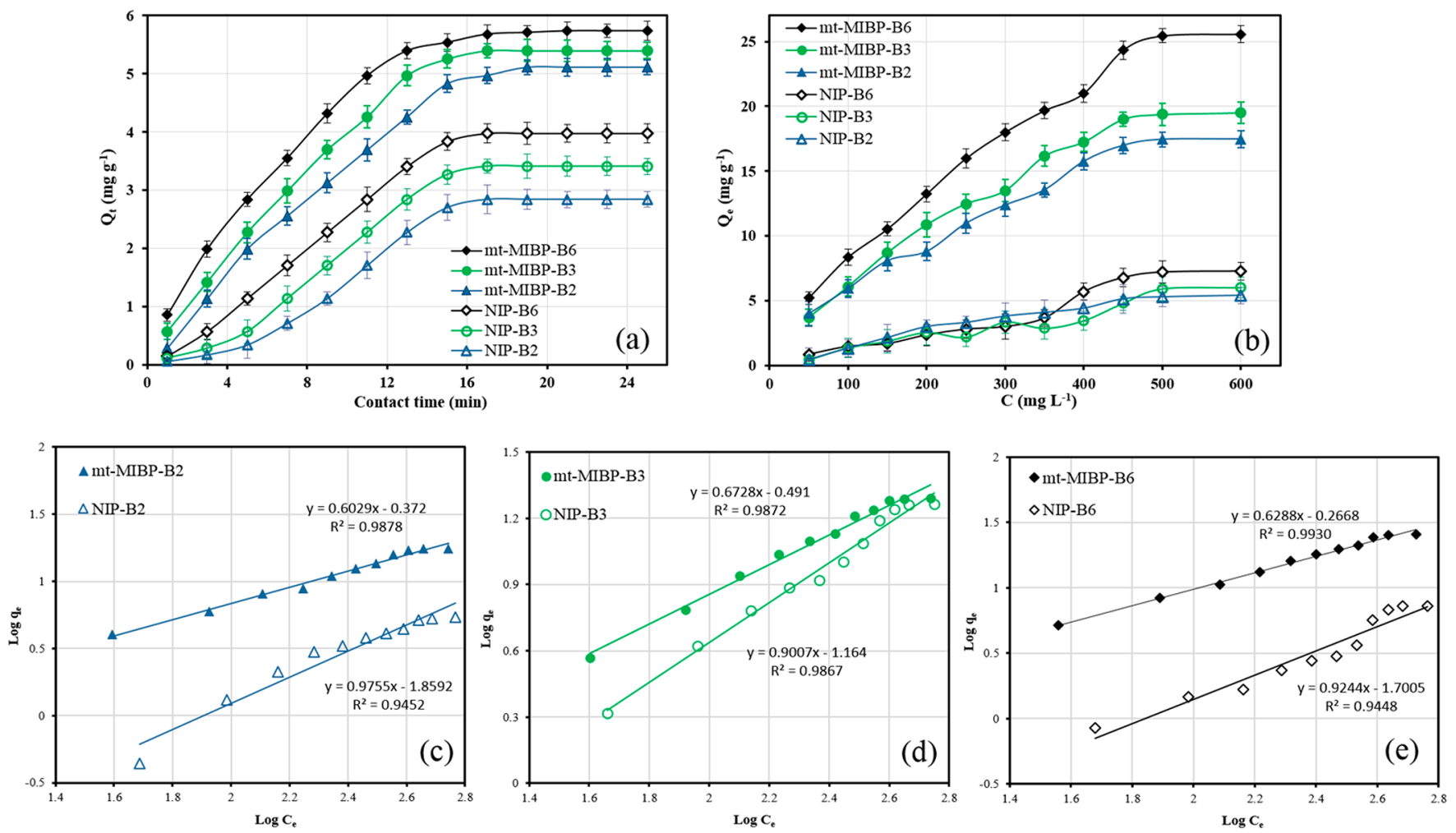

Figure 4. Adsorption kinetics (a) and isotherms (b) and related Freundlich fitting model curves (c-e) of mt-MIBP and NIP for the three template vitamins. 


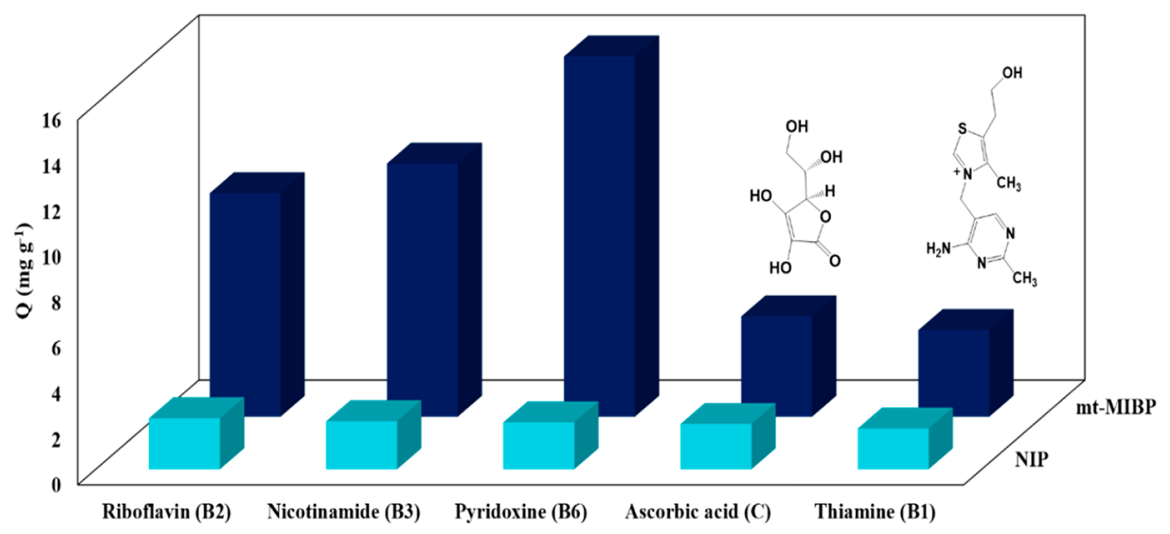

Figure 5. Adsorption capacity comparison of MIBP and NIP for the five vitamins. Experimental conditions: $40 \mathrm{mg}$ of mt-MIBP or NIP, concentration of vitamins at $250.0 \mathrm{mg} \mathrm{L}^{-1}$ individually.

some disadvantage of bulk imprinted polymers and nonthin materials. ${ }^{39}$ The adsorption kinetic data of mt-MIBP and NIP were fitted by pseudo-first-order and pseudo-second-order models. Related parameters are listed in Table S1, along with fitting curves in Figure S5. As seen, the pseudo-second-order model yielded a better fit, with correlation coefficients $\left(R^{2}\right)$ above 0.96 . Thus, the adsorption obeyed the pseudo-secondorder kinetics model.

The adsorption capacity is a substantial factor to evaluate the selective binding and recognition ability of imprinting effect on prepared materials. The adsorption isotherms of vitamins in the mt-MIBP and NIP in Figure $4 \mathrm{~b}$ revealed that the adsorption capacities increased by elevating the initial concentration and subsequently specific cavities became saturated at $500 \mathrm{mg} \mathrm{L}^{-1}$. The binding properties of the mt-MIBP and NIP were estimated by the Langmuir, Freundlich, Temkin, and Dubinin-Radushkevich isotherm models. According to related fitting parameters listed in Table S2, the Freundlich model was suitable. The estimation equation is as below

$$
\log Q=m \log C+\log \alpha
$$

where $Q\left(\mathrm{mg} \mathrm{g}^{-1}\right)$ is the amount of analytes adsorbed per mass unit of sorbents at the equilibrium state, $C\left(\mathrm{mg} \mathrm{L}^{-1}\right)$ is the equilibrium vitamins concentration in sample solution, $\alpha$ is the Freundlich constant indicating a measure of capacity and average affinity, and $m$ is the heterogeneity index which can take values from 1 to 0 , where the values of 1 and 0 refer to homogeneous and heterogeneous systems, respectively. Figure $4 c-d$ depicts the adsorption isotherms of three vitamins on the mt-MIBP and NIP fitting their experimental data by the Freundlich isotherm. As seen, linear correlation coefficients greater than 0.99 could strongly support the presence of heterogeneous binding sites in both sorbents structure which has good agreement with the results of the BET. In addition, our present results could well confirm the Freundlich isotherm model with unique ability to supply information about binding site homogeneity for most noncovalently imprinted polymers. ${ }^{40}$ The maximum adsorption capacities for $\mathrm{B}_{2}, \mathrm{~B}_{3}$, and $\mathrm{B}_{6}$, were attained of 17.4, 19.5, and $25.6 \mathrm{mg} \mathrm{g}^{-1}$ by mt-MIBP, respectively, while $5.4,6.0$, and $7.3 \mathrm{mg} \mathrm{g}^{-1}$ by NIP, respectively. These results demonstrated that the presence of all the templates during synthesis played a vital role in formation of specific cavities in the mt-MIBP skeleton. Furthermore, the difference in maximum adsorption capacities for different templates is very likely owing to the different structural formulas and intermolecular competition. ${ }^{6,23,36}$ Also, the phenomenon is generally present in multitemplate imprinting researches. $^{6,23,25,36,37}$

3.3. Selectivity Examination of mt-MIBP. In order to verify that mt-MIBP was selective for the three template vitamins, two different analogues of ascorbic acid (vitamin $\mathrm{C}$ ) and thiamine (vitamin $B_{1}$ ) were tested. As seen in Figure 5, the adsorption capacities for three templates were much higher than that for analogues, and experimental results suggested that mt-MIBP had satisfactory anti-interference ability. In addition, the variation in the molecular size and functional groups of vitamin $\mathrm{C}$ and vitamin $\mathrm{B}_{1}$ compared to all of the three template analytes hindered the accessibility of the imprinting sites, which led to significant reduction of sorption capacity. It should be noted that the selectivity order was $B_{6}>B_{3}>B_{2}$, which can be ascribed to the difference in their structure formula. ${ }^{6,23,36}$ As seen from Figure $\mathrm{S} 6, \mathrm{~B}_{6}$ has a more symmetrical molecular structure and offers four possible binding sites, leading to the preferential adsorption. Although $\mathrm{B}_{6}$ has more possible binding sites, its large molecule size and steric hindrance will greatly decrease its competitiveness among the three templates in one MIPs format. Overall, the results revealed that the completely matching in all the size, shape, functional groups, and stereochemistry made a significant contribution to the high selectivity of the mt-MIBP.

3.4. Condition Optimization for d-SPE of Vitamins in Orange Juice Samples. To attain maximum extraction efficiency of vitamins in orange juice samples, it was necessary to investigate the preliminary influences of main factors including sorbent dosage, initial $\mathrm{pH}$ of sample solution, sonication time, and kinds and volumes of washing and eluting solvents. A spiked concentration level of $200.0 \mu \mathrm{g} \mathrm{L}^{-1}$ of individual vitamins $\mathrm{B}$ was used.

Orange juice has a complicated matrix in which some interferents retained on mt-MIBP not only may have peaks overlapped with analytes peaks but also can lead to serious pollution of the analytical $\mathrm{C}_{18}$ HPLC column. To reduce such encountered problematic interfering species, performing a preliminary washing step has a crucial role to obtain clean extraction. For this aim, different solvents with various polarities such as deionized water, methanol, acetonitrile, acetone, and hexane were examined and their effects were investigated. Among tested solvents, the cleanest extracts were attained using hexane as washing solvent. Due to the hydrophilicity of mt-MIBP and the high potential of hexane to solve nonpolar interferents, the residuary of hydrophobic matrix interference is avoided, while the interactions between 
analytes and sorbent do not change significantly. In the next step, the effect of solvent volume was evaluated in the range of $1.0-5.0 \mathrm{~mL}$, and experimental results showed that $2.0 \mathrm{~mL}$ of hexane was sufficient to eliminate adverse matrix effects.

The selection of elution solvent is dependent on the vitamins' polarity since the target analytes should be completely eluted. The hydrophilic nature of all analytes encouraged us to apply different polar solvents with strong elution strength as well as changing their various volume ratios including methanol, acetonitrile, deionized water, methanol/acetonitrile (50:50, v/v), methanol/ammonium hydroxide (90:10, v/v), acetonitrile/ammonium hydroxide $(90: 10, \mathrm{v} / \mathrm{v})$, deionized water/ammonium hydroxide (90:10, v/v), and methanol/ acetonitrile/deionized water/ammonium hydroxide (30:30:30:10, v/v) were assessed. The results indicated that methanol/acetonitrile/deionized water/ammonium hydroxide $(30: 30: 30: 10, \mathrm{v} / \mathrm{v})$ was an appropriate eluent, in which most of analytes could be eluted from the mt-MIBP and the highest extraction efficiency was achieved, as shown in Figure S7. Indeed, methanol and acetonitrile are polar solvents which are able to break hydrogen bonds between vitamins and functional groups of chitosan in mt-MIBP. Moreover, the evaluation of the eluent volumes in the range of $1.0-5.0 \mathrm{~mL}$ showed that $3.0 \mathrm{~mL}$ of eluent was enough to desorb analytes completely.

3.5. Parameter Effects Evaluation via CCD and Response Surface. The optimization process has been often fulfilled using effective multivariate techniques. Accordingly, surface response methodologies (RSMs) have been adopted as proper alternatives and resulted in empirically polynomial relationships among target response and independent variables by spending minimum time and expense. ${ }^{41,42}$ Second-order models were more applicable, especially in the establishment of parabolic curvatures by consideration of significant quadratic and interactive effects. Consequently, maximum information for an intended process was easily achieved via these optimization procedures. ${ }^{43}$ In the current study, statistical evaluation through $\mathrm{CCD}$ for three main factors (initial sample $\mathrm{pH}$, sonication time, and sorbent dosage) affecting d-SPE of B-family vitamins from orange juice sample was performed. It consisted of 4 fractional factorial points, 6 axial points, and 5 central points (Table S3). Investigation of their significance was undertaken by analysis of variance (ANOVA) based on rejection of the null hypothesis via Fisher's tests (F-test). According to Table S4, higher Fvalues (or $P$-values lower than 0.05 at a specified confidence level $(\alpha=0.05)$ implied significance of each term. Established quadratic models with respect to the mentioned criteria for evaluation of the extraction recovery (ER (\%)) in terms of actual factors are shown in the Supporting Information.

As seen, robustness of these fitted models was checked via a lack-of-fit test; $P$-values higher than 0.05 confirmed it was not significant, while also, reliability was assayed through the multiple correlation coefficients which were higher than 0.90, indicating high accuracy of the constructed models. The main parameters as well as their influential interactions were studied in detail via response surface diagrams (Figure S8). The existence of semicurvature in 3D plots illustrated significance of interactive effects. Figure S8 displays the significant interactive effect of $\mathrm{BC}$ onto the extraction of $\mathrm{B}_{2}$ vitamin (namely, the interaction influence between sample $\mathrm{pH}$ and sonication time). As seen, by concurrent increment of initial $\mathrm{pH}$ medium and sonication time (especially $\mathrm{pH}$ value), the preconcentration of $\mathrm{B}_{2}$ vitamin was enhanced remarkably. The $\mathrm{pH}$ value played an impressive role in the sorption process. In this sense, the predominant forms of adsorbent/adsorbate and respective interaction type were extremely influenced by $\mathrm{pH}$. Chitosan has a $\mathrm{p} K_{\mathrm{a}}$ value of $\sim 6.5$, which leads to a protonation in acidic to neutral solution with a charge density dependent on $\mathrm{pH}$. On the other hand, the analyzed B-family vitamins have different acidic dissociation constant, i.e., 3.35 for $\mathrm{B}_{3}, 10.2$ for $\mathrm{B}_{2}$, and 5.6 and 8.6 for $\mathrm{B}_{6}$, which encourage researchers to select the proper $\mathrm{pH}$ value for achievement of best extraction efficiency. Consequently, in $\mathrm{pH}$ below $\mathrm{p} K_{a}$, vitamins have positive charges, and, in $\mathrm{pH}$ higher than $\mathrm{pK}_{\mathrm{a}}$ they are in natural forms. On other words, in acidic media, the $\mathrm{N}$ atoms incorporated in pyridine rings established coordinative linkages with hydronium ions and led to deteriorative interactions with the proposed sorbent and subsequently poor extraction efficiencies. However, the molecularly dominant species formed in basic media had effective affinity to connect with included cavities via two ways, viz. establishment of impressive hydrogen bonding (as shown in Figure S6) and the memory effects elaborated in polymeric network. As seen in Figure S8, the initial $\mathrm{pH}$ value had sharp influence onto $\mathrm{B}_{2}$ recovery and maximum efficiency was achieved at $\mathrm{pH} 11.0$ and moderate sonication time which confirm the above claim completely. Influential turbulent mixing induced by ultrasonic waves was also determinative in the attainment of maximum extraction efficiency (especially in the middle region) due to fine dispersion of solid phase in aqueous media, which provided fast and effective mass transfer of target analytes to sorbent within the cavitation phenomenon. As well, the figure shows the significant interaction effect of sonication time and adsorbent dosage $(\mathrm{AB})$ onto the extraction of $\mathrm{B}_{6}$ vitamin. As seen, with simultaneous increase of these main parameters, the extraction efficiency first enhances and then it remains constant. Also, the general direction of extraction recovery toward higher yields is influenced equivalently by both variations of sonication time and adsorbent dosage up to mediocre operational spans. These constructive effects can be justified by increment of the sorbent amount which supplies more specific surface areas which are applicable for trapping target analytes along fine dispersion induced via the cavitation phenomenon which total cause considerable improvement in the uptake of analyte of interest. The significant interactive effect of $\mathrm{AC}$ onto the extraction of $\mathrm{B}_{6}$ vitamin (namely, the interaction impact between sample $\mathrm{pH}$ and sorbent dosage) is also depicted in Figure S7. The effect of initial $\mathrm{pH}$ value is more considerable then sorbent dosage onto uptake of $\mathrm{B}_{6}$ vitamin. Diverse dissociation constants of $\mathrm{B}_{6}$ vitamin (5.6 and 8.6) encourage adjusting alkaline media to obtain maximum extraction of $\mathrm{B}_{6}$ vitamin, because the target analyte is uncharged and can form the considerable interaction with the imprinted cavities via hydrogen bonding and van der Waals forces. As shown, this positive effect is more significant at the relatively moderate values of sorbent dosage.

Consequently, the highest extraction recoveries (higher than $90 \%)$ were successfully obtainable at alkaline $\mathrm{pH}$ (11.0), $40 \mathrm{mg}$ of sorbent, and $20.0 \mathrm{~min}$ of sonication time. Desirability function (DF) values for the mentioned conditions were close to unit and replicate at the above specific conditions, which were checked to examine pure error and also check applicability of this optimum point for prediction of real behavior of the system. These optimized conditions were repeated three times. As seen from Table S4, the satisfactory compatibility between responses average and one predicted via DF certifies high adequacy of the developed models. 

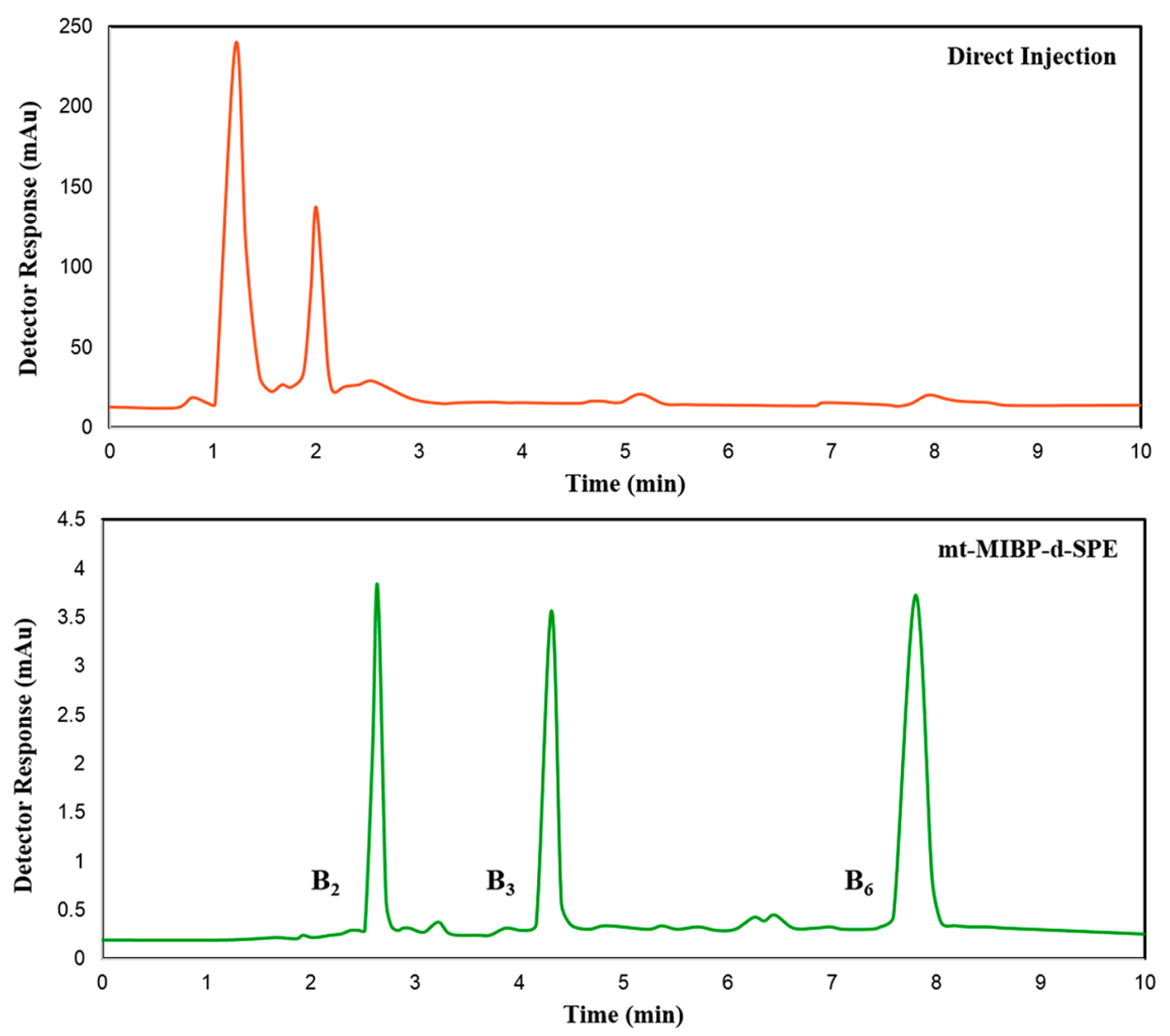

Figure 6. Typical chromatograms of direct injection of orange juice sample and orange juice sample after mt-MIBP-d-SPE procedure. Spiked concentrations: $\mathrm{B}_{2}, 300 \mu \mathrm{g} \mathrm{L}^{-1} ; \mathrm{B}_{3}, 200 \mu \mathrm{g} \mathrm{L}^{-1} ; \mathrm{B}_{6}, 50 \mu \mathrm{g} \mathrm{L}^{-1}$.

\subsection{Method Validation and Real Application of mt-} MIBP-d-SPE. It is well-known that detection at proper maximum wavelengths can lead to improvements in figures of merit like accuracy and sensitivity during the simultaneous determination of analytes by HPLC-UV. Deviation from optimal detection wavelengths causes occurrence of nondetected or false values in numerical value that correspond to trace detection and lead to discrepancies and incomparability of the results. Owning to respective characteristic structures of three vitamins and different absorptive intensity in UV, the proper detection wavelength should be set for every analyte to accurately quantify their contents. To overcome mentioned pitfalls, the effects of wavelengths changing in the range of 265-290 nm with $1 \mathrm{~nm}$ intervals on the peak area of the three vitamins were investigated. The representative chromatograms of the vitamin standards are shown in Figure S9. As can be seen, the optimized wavelengths for riboflavin, nicotinamide, and pyridoxine were 270, 274, and $284 \mathrm{~nm}$, respectively. Wavelength-switching detection gave more peak area of analytes to minimize the interferences from solvents and impurities with respect to constant wavelength.

The mt-MIBP-d-SPE-HPLC/UV method for the determination of B-family vitamins in orange juice was validated in terms of linearity range, limit of detection (LOD), limit of quantification (LOQ), robustness, and accuracy. The linearity of the extraction method was measured by plotting peak area versus analyte concentration to create the calibration curves. Wide linearity was obtained, over the range of $0.01-3.0 \mathrm{mg} \mathrm{L}^{-1}$ for $\mathrm{B}_{2}, 0.02-2.0 \mathrm{mg} \mathrm{L}^{-1}$ for $\mathrm{B}_{3}$, and $0.005-5.0 \mathrm{mg} \mathrm{L}^{-1}$ for $\mathrm{B}_{6}$, with correlation coefficients $\geq 0.99$, indicating excellent linearity. The LODs and LOQs, defined as $3 \sigma$ /slope and 10 $\sigma /$ slope ratios, respectively, where $\sigma$ is the standard deviation for 3 and 10 chromatograms obtained from the blank, ${ }^{44}$ were 2.8 and $9.3 \mu \mathrm{g} \mathrm{L}^{-1}$ for riboflavin, 5.5 and $18.4 \mu \mathrm{g} \mathrm{L}^{-1}$ for nicotinamide, and 1.2 and $4.0 \mu \mathrm{g} \mathrm{L}^{-1}$ for pyridoxine, which confirmed good operational condition and merit of the present method. The precision, reproducibility, and repeatability (intraday and inter-day coefficients of variation) of the method are listed in Table S5. As seen, the values of RSDs $\leq 4.2$ of intraday and inter-day precisions were satisfactory, which supported qualified reproducibility of the present method.

The accuracy and potential application of the mt-MIBP-dSPE-HPLC/UV method for selective purification and preconcentration of vitamins in three orange juice samples were checked. As shown in Figure 6, for direct injection of a crude orange juice sample, quite wide and high impurity peaks appeared while no vitamin peaks were found, which indicated endogenous vitamins could not be detected by HPLC without the sample preparation step because of the impurity interferences. Happily, preconcentration and cleanup via mtMIBP-d-SPE led to dominant vitamins peaks in HPLC chromatograms, while significantly eliminating impurities signal (Figure 6). Accuracy was determined through evaluation of the recovery obtained at four different concentrations using a standard addition method. As listed in Table 1, the RR\% values were found to be in the range of $75.8-92.7 \%$ for riboflavin, 81.1-92.5\% for nicotinamide, and $84.7-93.8 \%$ for pyridoxine with relative standard deviations (RSDs, \%) lower than 4.0, by using three continuous injections of four concentrations. These superior results confirmed that the developed mt-MIBP-d-SPE is suitable for efficient enrichment and purification of vitamins from the complex orange juice samples. 


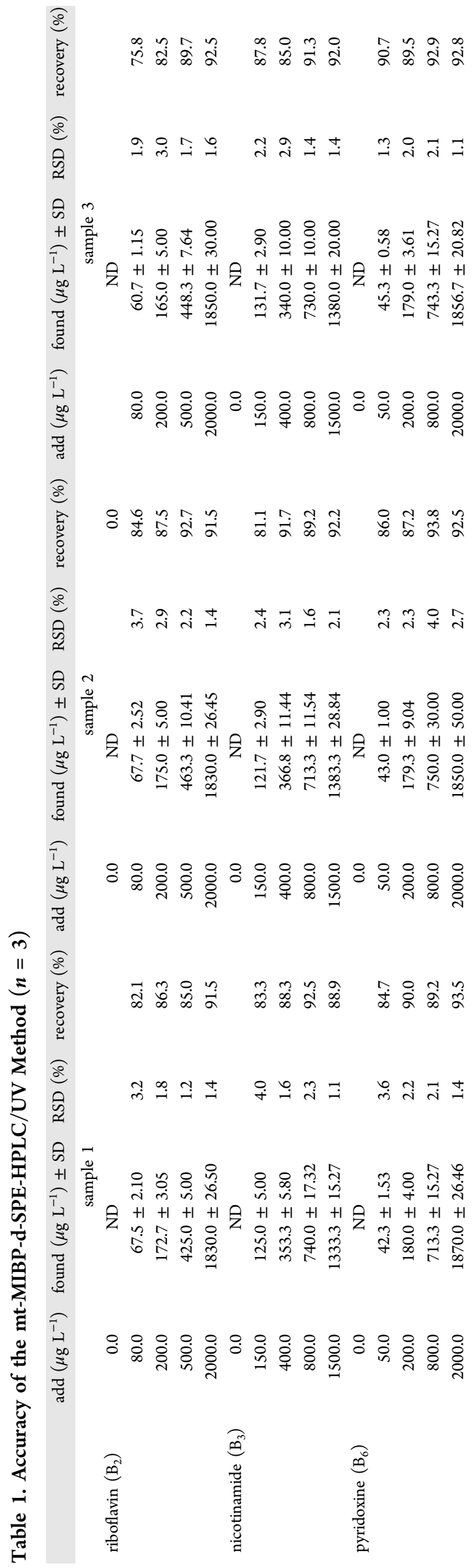

A typical comparison between different methods for determination of $\mathrm{B}$ vitamins is summarized in Table S6. ${ }^{26-28,45-51}$ As seen, the obtained linear range and LOD value of the present approach are comparable with those of other research works. Comparing with the highly sensitive, complex, and expensive MS-based methods, the LOD value is satisfactory, proving that the pretreatment in this work was much efficient and highly selective, followed by the relatively simple and cheap HPLC-UV technique. Furthermore, although in ref 50 fluorescence detection provides higher selectivity and sensitivity in comparison with UV detection, the linear range of the current work is wider, which is mainly attributed to unique advantages of the present selective and sensitive sample preparation step to eliminate matrix interferences. Through the comparison, supreme capability of noble mt-MIBP in clean preconcentration of water-soluble vitamins in orange juice samples at trace level was certified.

\section{CONCLUSIONS}

In summary, new mt-MIBP with good water compatibility and multiple recognition sites was smartly prepared based on a green synthesis strategy via the synergy of CSs and chitosan with less consumption of organic solvents by combining surface imprinting and multitemplate imprinting approaches. The mtMIBP was successfully applied for simultaneously selective dSPE of three applicable B-family vitamins from orange juice samples, followed by HPLC determination. The synthesis route possessed the impressive merits of no toxicity, less consumption of organic solvent, affordability, biosource reagents, waste prevention, at the least amount of glassware, and environmentally friendly reaction conditions. By embedding CSs into the imprinted chitosan shell, the mt-MIBP acquired high binding capacity and fast kinetics, which facilitated high detection sensitivity and excellent aqueous applicability. Besides, an experimental design approach of CCD with response surface proved a good alternative for multivariable optimization. Furthermore, high throughput mt-MIPsbased eco-friendly platforms can be expected for selectively concurrent quantification of water-soluble compounds in various complicated matrixes, by making more efforts to continuously improve the imprinting performances of mt-MIPs, as well as by rationally utilizing diverse facile functionalization and interactions (from CS (from glucose), chitosan, etc.) for green synthesis to satisfy sustainable development.

\section{ASSOCIATED CONTENT}

\section{Supporting Information}

The Supporting Information is available free of charge on the ACS Publications website at DOI: 10.1021/acsami.7b17500.

Related descriptions about influence factors examination and extraction recovery (ER), SEM/TEM images, size distribution profile, nitrogen adsorption/desorption (ADS/DES) isotherms, kinetic fitting curves, hydrogen bonding formation schematic, eluent solvent effect, response surface diagrams, chromatograms of vitamin standard solutions, adsorption kinetic model fitting parameters, adsorption isotherm fitting parameter, CCD with experimental results, analysis of variance, precision of the MTMIBP-d-SPE-HPLC/UV method, method performance comparison (PDF) 


\section{AUTHOR INFORMATION}

\section{Corresponding Authors}

*E-mail: m_ghaedi@mail.yu.ac.ir. Tel/Fax: +98-741-2223048 (M.G.).

*E-mail: lxchen@yic.ac.cn. Tel/Fax: +86 5352109130 (L.C.).

\section{ORCID $\odot$}

Maryam Arabi: 0000-0002-2028-9572

Lingxin Chen: 0000-0002-3764-3515

\section{Notes}

The authors declare no competing financial interest.

\section{ACKNOWLEDGMENTS}

This work was financially supported by the Graduate School and Research Council of Yasouj University, the National Natural Science Foundation of China (21477160, 41776110), and the National Defense Science and Technology Innovation Project of Chinese Academy of Sciences (CXJJ-16M254).

\section{REFERENCES}

(1) Ruiz-Aceituno, L.; Carrero-Carralero, C.; Ruiz-Matute, A. I.; Ramos, L.; Sanz, M. L.; Martínez-Castro, I. Characterization of Cyclitol Glycosides by Gas Chromatography Coupled to Mass Spectrometry. J. Chromatogr. A 2017, 1484, 58-64.

(2) Wen, Y.; Chen, L.; Li, J.; Liu, D.; Chen, L. Recent Advances in Solid-Phase Sorbents for Sample Preparation Prior to Chromatographic Analysis. TrAC, Trends Anal. Chem. 2014, 59, 26-41.

(3) de Faria, H. D.; de Carvalho Abrão, L. C.; Santos, M. G.; Barbosa, A. F.; Figueiredo, E. C. New Advances in Restricted Access Materials for Sample Preparation: A Review. Anal. Chim. Acta 2017, 959, 43-65.

(4) Arabi, M.; Ghaedi, M.; Ostovan, A. Development of a Lower Toxic Approach Based on Green Synthesis of Water-Compatible Molecularly Imprinted Nanoparticles for the Extraction of Hydrochlorothiazide from Human Urine. ACS Sustainable Chem. Eng. 2017, 5, 3775-3785.

(5) Yang, Y.; Niu, H.; Zhang, H. Direct and Highly Selective Drug Optosensing in Real, Undiluted Biological Samples with QuantumDot-Labeled Hydrophilic Molecularly Imprinted Polymer Microparticles. ACS Appl. Mater. Interfaces 2016, 8, 15741-15749.

(6) Chen, L.; Wang, X.; Lu, W.; Wu, X.; Li, J. Molecular Imprinting: Perspectives and Applications. Chem. Soc. Rev. 2016, 45, 2137-2211.

(7) Ostovan, A.; Ghaedi, M.; Arabi, M.; Asfaram, A. Hollow Porous Molecularly Imprinted Polymer for Highly Selective Clean-up Followed by Influential Preconcentration of Ultra-trace Glibenclamide from Bio-fluid. J. Chromatogr. A 2017, 1520, 65-74.

(8) Yang, R.; Liu, Y.; Yan, X.; Liu, S.; Zheng, H. An Effective Method for the Synthesis of Yolk-Shell Magnetic Mesoporous Carbon-Surface Molecularly Imprinted Microspheres. J. Mater. Chem. A 2016, 4, 9807-9815.

(9) Fang, Y.; Gu, D.; Zou, Y.; Wu, Z.; Li, F.; Che, R.; Deng, Y.; Tu, B.; Zhao, D. A Low Concentration Hydrothermal Synthesis of Biocompatible Ordered Mesoporous Carbon Nanospheres with Tunable and Uniform Size. Angew. Chem., Int. Ed. 2010, 49, 79877991.

(10) Qin, L.; Jia, X.; Yang, Y.; Liu, X. Porous Carbon Microspheres: An Excellent Support To Prepare Surface Molecularly Imprinted Polymers for Selective Removal of Dibenzothiophene in Fuel Oil. Ind. Eng. Chem. Res. 2016, 55, 1710-1719.

(11) Liu, W.; Qin, L.; Shi, W.; Chen, L.; Yang, Y.; Liu, X.; Xu, B. Molecularly Imprinted Polymers on the Surface of Porous Carbon Microspheres for Capturing Dibenzothiophene. Microchim. Acta 2016, $183,1153-1160$.

(12) Liu, W.; Zhao, H.; Yang, Y.; Liu, X.; Xu, B. Reactive Carbon Microspheres Prepared by Surface-Grafting 4-(Chloromethyl) Phenyltrimethoxysilane for Preparing Molecularly Imprinted Polymer. Appl. Surf. Sci. 2013, 277, 146-154.
(13) Zheng, H.; Yoshikawa, M. Molecularly Imprinted Cellulose Membranes for Pervaporation Separation of Xylene Isomers. J. Membr. Sci. 2015, 478, 148-154.

(14) Khorrami, A. R.; Rashidpur, A. Design of a New Cartridge for Selective Solid Phase Extraction Using Molecularly Imprinted Polymers: Selective Extraction of Theophylline from Human Serum Samples. Biosens. Bioelectron. 2009, 25, 647-651.

(15) Shen, X.; Xu, C.; Ye, L. Molecularly Imprinted Polymers for Clean Water: Analysis and Purification. Ind. Eng. Chem. Res. 2013, 52, 13890-13899.

(16) Dirion, B.; Cobb, Z.; Schillinger, E.; Andersson, L. I.; Sellergren, B. Water-Compatible Molecularly Imprinted Polymers Obtained via High-Throughput Synthesis and Experimental Design. J. Am. Chem. Soc. 2003, 125, 15101-15109.

(17) Yang, S.; Qian, J.; Kuang, L.; Hua, D. Ion-Imprinted Mesoporous Silica for Selective Removal of Uranium from Highly Acidic and Radioactive Effluent. ACS Appl. Mater. Interfaces 2017, 9, 29337-29344.

(18) Liu, J. Q.; Wulff, G. N. Functional Mimicry of Carboxypeptidase A By a Combination of Transition State Stabilization and a Defined Orientation of Catalytic Moieties in Molecularly Imprinted Polymers. J. Am. Chem. Soc. 2008, 130, 8044-8054.

(19) Helling, S.; Shinde, S.; Brosseron, F.; Schnabel, A.; Müller, T.; Meyer, H. E.; Marcus, K.; Sellergren, B. R. Ultratrace Enrichment of Tyrosine Phosphorylated Peptides on an Imprinted Polymer. Anal. Chem. 2011, 83, 1862-1865.

(20) Yang, K.; Berg, M. M.; Zhao, C.; Ye, L. One-Pot Synthesis of Hydrophilic Molecularly Imprinted Nanoparticles. Macromolecules 2009, 42, 8739-8746.

(21) Hoshina, K.; Horiyama, S.; Matsunaga, H.; Haginaka, J. Molecularly Imprinted Polymers for Simultaneous Determination of Antiepileptics in River Water Samples by Liquid ChromatographyTandem Mass Spectrometry. J. Chromatogr. A 2009, 1216, 49574962.

(22) Arabi, M.; Ghaedi, M.; Ostovan, A. Water Compatible Molecularly Imprinted Nanoparticles as a Restricted Access Material for Extraction of Hippuric Acid, a Biological Indicator of Toluene Exposure, from Human Urine. Microchim. Acta 2017, 184, 879-887.

(23) Song, X.; Li, J.; Xu, S.; Ying, R.; Ma, J.; Liao, C.; Liu, D.; Yu, J.; Chen, L. Determination of 16 Polycyclic Aromatic Hydrocarbons in Seawater Using Molecularly Imprinted Solid-Phase Extraction Coupled with Gas Chromatography-Mass Spectrometry. Talanta 2012, 99, 75-82.

(24) Madikizela, L. M.; Chimuka, L. Determination of Ibuprofen, Naproxen and Diclofenac in Aqueous Samples Using a MultiTemplate Molecularly Imprinted Polymer as Selective Adsorbent for Solid-Phase Extraction. J. Pharm. Biomed. Anal. 2016, 128, 210-215.

(25) Lu, W.; Wang, X.; Wu, X.; Liu, D.; Li, J.; Chen, L.; Zhang, X. Multi-Template Imprinted Polymers for Simultaneous Selective SolidPhase Extraction of Six Phenolic Compounds in Water Samples Followed by Determination Using Capillary Electrophoresis. J. Chromatogr. A 2017, 1483, 30-39.

(26) Chen, Z.; Chen, B.; Yao, S. High-Performance Liquid Chromatography/Electrospray Ionization-Mass Spectrometry for Simultaneous Determination of Taurine and 10 Water-Soluble Vitamins in Multivitamin Tablets. Anal. Chim. Acta 2006, 569, 169175.

(27) Márquez-Sillero, I.; Cárdenas, S.; Valcárcel, M. Determination of Water-Soluble Vitamins in Infant Milk and Dietary Supplement Using a Liquid Chromatography On-Line Coupled to a Corona-Charged Aerosol Detector. J. Chromatogr. A 2013, 1313, 253-258.

(28) Marti-Andres, P.; Escuder-Gilabert, L.; Martin-Biosca, Y.; Sagrado, S.; Medina-Hernandez, M. Simultaneous Determination of Pyridoxine and Riboflavin in Energy Drinks by High-Performance Liquid Chromatography with Fluorescence Detection. J. Chem. Educ. 2015, 92, 903-906.

(29) Titirici, M. M.; Antonietti, M. Chemistry and Materials Options of Sustainable Carbon Materials Made by Hydrothermal Carbonization. Chem. Soc. Rev. 2010, 39, 103-116. 
(30) Zang, L.; Qiu, J.; Wu, X.; Zhang, W.; Sakai, E.; Wei, Y. Preparation of Magnetic Chitosan Nanoparticles as Support for Cellulase Immobilization. Ind. Eng. Chem. Res. 2014, 53, 3448-3454.

(31) Kuo, C. H.; Liu, Y. C.; Chang, C. M. J.; Chen, J. H.; Chang, C.; Shieh, C. J. Optimum Conditions for Lipase Immobilization on Chitosan-Coated $\mathrm{Fe}_{3} \mathrm{O}_{4}$ Nanoparticles. Carbohydr. Polym. 2012, 87, $2538-2545$.

(32) Cui, Z.; Xiang, Y.; Si, J.; Yang, M.; Zhang, Q.; Zhang, T. Ionic Interactions Between Sulfuric Acid and Chitosan Membranes. Carbohydr. Polym. 2008, 73, 111-116.

(33) Lee, Y. M.; Nam, S. Y.; Woo, D. J. Pervaporation of ionically surface crosslinked chitosan composite membranes for water-alcohol mixtures. J. Membr. Sci. 1997, 133, 103-110.

(34) Sevilla, M.; Fuertes, A. B. Chemical and Structural Properties of Carbonaceous Products Obtained by Hydrothermal Carbonization of Saccharides. Chem. - Eur. J. 2009, 15, 4195-4203.

(35) Nair, M. B.; Baranwal, G.; Vijayan, P.; Keyan, K. S.; Jayakumar, R. Composite Hydrogel of Chitosan-Poly (Hydroxybutyrate-CoValerate) with Chondroitin Sulfate Nanoparticles for Nucleus Pulposus Tissue Engineering. Colloids Surf., B 2015, 136, 84-92.

(36) Madikizela, L. M.; Mdluli, P. S.; Chimuka, L. Experimental and Theoretical Study of Molecular Interactions Between 2-Vinyl Pyridine and Acidic Pharmaceuticals Used as Multi-Template Molecules in Molecularly Imprinted Polymer. React. Funct. Polym. 2016, 103, 3343.

(37) Dai, C. M.; Zhang, J.; Zhang, Y. L.; Zhou, X. F.; Duan, Y. P.; Liu, S. G. Selective Removal of Acidic Pharmaceuticals from Contaminated Lake Water Using Multi-Templates Molecularly Imprinted Polymer. Chem. Eng. J. 2012, 211-212, 302-309.

(38) Mehdinia, A.; Kayyal, T. B.; Jabbari, A.; Aziz-Zanjani, M. O.; Ziaei, E. Magnetic Molecularly Imprinted Nanoparticles Based on Grafting Polymerization for Selective Detection of 4-Nitrophenol in Aqueous Samples. J. Chromatogr. A 2013, 1283, 82-88.

(39) Kong, X.; Gao, R.; He, X.; Chen, L.; Zhang, Y. Synthesis and Characterization of the Core-Shell Magnetic Molecularly Imprinted Polymers $\left(\mathrm{Fe}_{3} \mathrm{O}_{4} @ M I P s\right)$ Adsorbents for Effective Extraction and Determination of Sulfonamides in the Poultry Feed. J. Chromatogr. A 2012, 1245, 8-16.

(40) Umpleby, R. J.; Baxter, S. C.; Bode, M.; Berch, J. K.; Shah, R. N.; Shimizu, K. D. Application of the Freundlich Adsorption Isotherm in the Characterization of Molecularly Imprinted Polymers. Anal. Chim. Acta 2001, 435, 35-42.

(41) Arabi, M.; Ostovan, A.; Ghaedi, M.; Purkait, M. K. Novel Strategy for Synthesis of Magnetic Dummy Molecularly Imprinted Nanoparticles Based on Functionalized Silica as an Efficient Sorbent for the Determination of Acrylamide in Potato Chips: Optimization by Experimental Design Methodology. Talanta 2016, 154, 526-532.

(42) Ma, J.; Jiang, L.; Wu, G.; Xia, Y.; Lu, W.; Li, J.; Chen, L. Determination of Six Sulfonylurea Herbicides in Environmental Water Samples by Magnetic Solid-Phase Extraction Using Multi-Walled Carbon Nanotubes as Adsorbents Coupled with High-Performance Liquid Chromatography. J. Chromatogr. A 2016, 1466, 12-20.

(43) Wen, Y.; Li, J.; Yang, F.; Zhang, W.; Li, W.; Liao, C.; Chen, L. Salting-Out Assisted Liquid-Liquid Extraction with the Aid of Experimental Design for Determination of Benzimidazole Fungicides in High Salinity Samples by High-Performance Liquid Chromatography. Talanta 2013, 106, 119-126.

(44) Analytical Methods Committee. Recommendations for the Definition, Estimation and Use of the Detection Limit. Analyst 1987, 112, 199-204. DOI: 10.1039/an9871200199.

(45) Ciulu, M.; Solinas, S.; Floris, I.; Panzanelli, A.; Pilo, M. I.; Piu, P. C.; Spano, N.; Sanna, G. RP-HPLC Determination of Water-Soluble Vitamins in Honey. Talanta 2011, 83, 924-929.

(46) Karimian, N.; Zavar, M. H. A.; Chamsaz, M.; Ashraf, N.; Turner, A. P.; Tiwari, A. A Potential-Gated Molecularly Imprinted Smart Electrode for Nicotinamide Analysis. RSC Adv. 2015, 5, 35089-35096.

(47) Jin, P.; Xia, L.; Li, Z.; Che, N.; Zou, D.; Hu, X. Rapid Determination of Thiamine, Riboflavin, Nniacinamide, Pantothenic Acid, Pyridoxine, Folic Acid and Ascorbic Acid in Vitamins with
Minerals Tablets by High-Performance Liquid Chromatography with Diode Array Detector. J. Pharm. Biomed. Anal. 2012, 70, 151-157.

(48) Okamoto, H.; Nakajima, T.; Ito, Y. Simultaneous Determination of Water-Soluble Vitamins in a Vitamin-Enriched Drink by an InCapillary Enzyme Reaction Method. J. Chromatogr. A 2003, 986, 153161.

(49) Vidović, S.; Stojanović, B.; Veljković, J.; Pražić-Arsić, L.; Roglić, G.; Manojlović, D. Simultaneous Determination of Some WaterSoluble Vitamins and Preservatives in Multivitamin Syrup by Validated Stability-Indicating High-Performance Liquid Chromatography Method. J. Chromatogr. A 2008, 1202, 155-162.

(50) Zafra-Gómez, A.; Garballo, A.; Morales, J. C.; García-Ayuso, L. E. Simultaneous Determination of Eight Water-Soluble Vitamins in Supplemented Foods by Liquid Chromatography. J. Agric. Food Chem. 2006, 54, 4531-4536.

(51) Zand, N.; Chowdhry, B. Z.; Pullen, F. S.; Snowden, M. J.; Tetteh, J. Simultaneous Determination of Riboflavin and Pyridoxine by UHPLC/LC-MS in UK Commercial Infant Meal Food Products. Food Chem. 2012, 135, 2743-2749. 